\title{
EL MÉTODO DE COSTOS ABC Y SU APLICACIÓN EN LA UNIVERSIDAD NACIONAL DE CHIMBORAZO
}

\author{
THE ABC COST METHOD AND ITS APPLICATION IN THE NATIONAL UNIVERSI- \\ TY OF CHIMBORAZO
}

Otto Eulogio Arellano Cepeda Universidad Nacional de Chimborazo Riobamba, Ecuador ORCID: http://orcid.org/0000-0001-9580-1625 Correo electrónico: oarellano@unach.edu.ec

\section{RESUMEN}

Objetivo: Determinar si el método de Costeo Basado en Actividades $(A B C)$ puede ser aplicado para construir el costo real de los servicios académicos en la Universidad Nacional de Chimborazo de la República del Ecuador. Método: Se aplicó el método inductivo, con un nivel descriptivo - causal. Se empleó el cuestionario para la obtención de información a una muestra de 56 trabajadores entre docentes y administrativos de una población total de 745 personas. Resultados: El análisis factorial y de regresión lineal muestran que existen procedimientos y actividades relacionados a la docencia, investigación y vinculación muy significativas que deben ser consideradas en la aplicación del método de costo $A B C$ en la Universidad. Conclusiones: El método de Costeo Basado en Actividades $(A B C)$ puede considerarse como un modelo eficiente que permite conocer los costos reales de los servicios académicos que oferta la Universidad y permite la toma de decisiones correctivas y de inversión en la mejora de la calidad educativa; además puede ser aplicado en todas las Instituciones de Educación Superior del Ecuador y de carácter educativo.

Palabras clave: Costos; sistema; actividades; contabilidad; gestión; educación.

\begin{abstract}
Objective: To determine how the Activity Based Costing (ABC) method and its application work in determining the real cost of education at the National University of Chimborazo of the Republic of Ecuador. Method: The inductive method is used, at a descriptive - causal level. The survey was applied to obtain the information of a sample of 56 workers between teachers and administrators staff of a total population of 745 people. Results: The results show that the application of a cost-per-activity system reduces procedures and improves accounting of the activities related to management, teaching and research in an appropriate manner. It is an efficient model that can be applied in Higher Education Institutions. Conclusions: The Activity-Based Costs method (ABC) can be consider as an efficient model that allows to know the real costs of the academic services offered by the University and allows the making of corrective and investment decisions in the improvement of the educational quality; Also can be applied in all Higher Education Institutions of Ecuador and Educational character.
\end{abstract}

Keywords: Costs; system; activities; accounting; management; education. 


\section{INTRODUCCIÓN}

Implementar un Sistema de Costos Basado en Actividades (Activity Based Costing o ABC) como un instrumento que permita la determinación del costo de los servicios de educación en las Instituciones de Educación Superior (IES), y que sirva como una herramienta para la toma de decisiones es importante; así como García, Marín y Martínez (2006) manifiestan que "los sistemas de contabilidad de costos deberían constituir herramientas comunes en los sistemas organizativos de todas las empresas con independencia de su tamaño" (p. 40). Según García (2008) "el sistema de costeo consiste fundamentalmente en asignar costos a los insumos necesarios para ejecutar todas las actividades de un proceso productivo" (p. 46). Desde esta perspectiva, en la Universidad Nacional de Chimborazo (UNACH) se observa que no se usa un sistema de contabilidad de costos definido que permita generar información adecuada y oportuna para tomar decisiones relacionadas al uso y administración del presupuesto institucional. En tal sentido, la investigación nace del siguiente cuestionamiento: ¿es posible que el sistema de costos por actividad permita determinar el costo real de los servicios académicos que oferta la Universidad Nacional de Chimborazo? ¿Cuáles son los procedimientos y actividades que debe considerarse para determinar los costos reales a través de la aplicación del costeo por actividades en la UNACH?

Las IES en el Ecuador y la Universidad Nacional de Chimborazo (UNACH), se encuentran reguladas por las siguientes normativas: Constitución de la República del Ecuador (2008), Ley Orgánica de Educación Superior (LOES), Reglamento a la Ley orgánica de Educación Superior, Reglamento de Régimen Académico CES, Estatuto Universitario, Reglamento de Carrera y Escalafón del Docente Investigador del Sistema de Educación Superior, y otras leyes conexas, reglamentos y disposiciones emanadas, por los órganos de control, como el Consejo de Educación Superior (CES) y por las normas internas de las Instituciones de Educación Superior.

Los modelos de costos en la universidad ecuatoriana se han enfocado en el cumplimiento y entrega de informes internos y externos solicitados por los órganos de control, y en las necesidades y regulaciones de la generación de informes contables, que es información insuficiente para los usuarios. Asimismo, se han enfocado en la determinación de costos de manera arbitraria sin responder a la realidad de las carreras, de las Facultades y del presupuesto institucional.

La determinación de los costos de los servicios que ofertan las IES es una variable que los administradores tienen bajo su control, las regulaciones establecidas de la gratui- dad de la educación superior hasta tercer nivel, y la regulación de los aranceles en las IES públicas y privadas por parte del CES afecta directamente al presupuesto de las instituciones, lo cual disminuye los ingresos y aumenta los gastos de operación.

Estudios realizados sobre la aplicación de costos $\mathrm{ABC}$ para las IES son muy pocos en la República del Ecuador, sin embargo, existen varios trabajos de investigación a nivel de América Latina y de Europa.

En tal sentido, tras una revisión bibliográfica se puede identificar que la aplicación de los costos $A B C$ se da en diferentes áreas de la industrias manufacturera (García, Marín y Martínez, 2006); asimismo, el sistema ABC se empleó en las empresas del sector privado, en seguida, fue adaptado en las organizaciones del sector público, principalmente en hospitales (Navarro, Martínez, Castilla y Hernández, 2006), (Fernandes, Barbosa, Mendes y Del Canto, 2014) y, posteriormente, en la educación, fue aplicado en las universidades, como muestran los estudios realizados por (López y Rodríguez, 2018; Boschin y Metz ,2009; Ficco , 2011; Pérez, Vergara, Moreno, Vergara y Rodríguez, 2008; Del Carpio, 2007) que han ayudado al progreso de procesos, dado que aumentan la fiabilidad de la información sobre los costos.

En este contexto, el objetivo de la investigación es determinar si el sistema de costos por actividad puede proporcionar los costos reales de servicios educativos en la Universidad Nacional de Chimborazo de la República del Ecuador en el periodo 2015-2016. Para ello se propone la siguiente $\mathrm{H} 1$ : El sistema de costos ABC influye significativamente en determinar los costos reales en la Universidad Nacional de Chimborazo, y la H2: Los procedimientos y actividades relacionados a de gestión, docencia e investigación influyen significativamente en la determinación de los costos reales en la Universidad Nacional de Chimborazo.

La elaboración de esta investigación se justifica porque distintos autores como (Ficco, 2011; Del Carpio, 2007; Tinjaca, 2005; López y Rodríguez, 2018) dan importancia a la contabilidad interna que se refiere al ciclo técnico de la corriente de la riqueza de la empresa en la cual tiene lugar el proceso de producción mediante la elaboración de las materias primas; asimismo se puede señalar que la contabilidad de costos es importante y es necesaria porque Arellano, Quispe, Ayaviri y Escobar (2017) definen que:

Los procedimientos contables y aún estadísticos dirigidos al conocimiento del costo en sus distintos estratos $y$ manifestaciones en las diferentes empresas. En este sentido se puede identificar que la contabilidad de costos no solamente se encarga de determinar el costo de producción (Materia Prima; Mano de Obra y Cos- 
tos Indirectos de Fabricación) sino también de llevar una estadística de la cantidad de bienes producidos y su capacidad instalada de producción (p. 34).

Además, García (2008) refiere que "la contabilidad de costos es un sistema de información empleado para predeterminar, registrar, acumular, controlar, analizar, direccionar, interpretar e informar todo lo relacionado con los costos de producción, venta, administración y financiamiento" (p. 8).

De esta manera, el diseño de un sistema de información de costos, busca llegar a cumplir con los objetivos relacionados con el cálculo de los costos de producción de bienes y servicios, como las que plantean los autores Horngren, Datar y Rajan (2012):

a) Generar información para ayudar en la planeación, evaluación y control de las operaciones de la empresa, b) Determinar los costos unitarios para evaluar los inventarios de producción en proceso y de artículos terminados, c) Generar informes sobre el costo de los artículos vendidos para determinar las utilidades, d) Contribuir a la planeación de utilidades proporcionando anticipadamente, e) Los costos de producción, distribución, administración y financiamiento, f) Contribuir en la elaboración de los presupuestos de la empresa, programas de producción, ventas y financiamiento (p. 35).

Una de las herramientas para el progreso de un sistema de costeo $\mathrm{ABC}$ es el diseño de los procesos y la identificación de las actividades individuales que consumen recursos y que son el objeto de acumulación de los costos. Primero, los procesos se plantean y, posteriormente, se diseñan; estos, continuamente, se mejorarán con el avance de la ciencia y la tecnología, se rediseñarán de acuerdo con las necesidades productivas, o cuando ya han cumplido su ciclo de vida.

Los procesos que aportan a la generación de valor según Uribe (2011) son: "los que agregan valor, los facilitadores o de apoyo y los administradores de activos" (p. 223); un proceso de costos ABC aplicado a las IES es una actividad interrelacionada que consume recursos para la ejecución de una tarea, o una unidad de trabajo compuesta de personas, tecnología, materias primas y métodos, para la producción de un bien o servicio.

\section{MATERIAL Y MÉTODOS}

Se aplicó el método inductivo, con un nivel descriptivo-causal. El diseño de la investigación es de tipo no experimental, usa un enfoque cualitativo y cuantitativo. Se utiliza un cuestionario como instrumento para la recolección de información. La muestra estuvo constituida por 56 trabajadores entre docentes y administrativos de una población total de 745 personas. La población fue estratificada considerando los departamentos (estratos): la gestión, docencia, investigación y vinculación; identificándose que la población en cada estrato es menor a 100 trabajadores, con excepción del estrato docencia, que alcanzó una población total de 694 docentes a los cuales se aplicó el método Delphi. Dado que fue importante la opinión de expertos para la identificación de procesos y actividades que permitan diseñar un modelo de costos $\mathrm{ABC}$, se eligió 14 docentes, 10 de ellos eran titulares a tiempo completo y los otros 4 docentes eran investigadores a tiempo completo no titulares de la Facultad de Ciencias Políticas y Administrativas.

Los datos fueron analizados con el uso de la estadística descriptiva y la multivariante, a través de la regresión lineal y la factorización y el análisis de correlación a datos cuantitativos, para dar respuesta a los objetivos y la comprobación de la hipótesis, considerando que el cuestionario tiene un grado de confiabilidad de 95\% (KMO = 0,95).

\section{RESULTADOS}

La Universidad Nacional de Chimborazo de acuerdo al Estatuto de la Universidad (2103) con Resolución RPCSO-36-No. 373-2013 y el Plan estratégico de desarrollo Institucional (2012-2016) resolución Nro. 0337-HCU-1710-2012 es reconocida como:

Una institución de educación superior, con personería jurídica, sin fines de lucro, autónoma, de derecho público, creada mediante Ley No. 98, publicada en el Suplemento del Registro Oficial No. 771, del 31 de agosto de 1995, su domicilio principal es la ciudad de Riobamba; sus siglas son UNACH. Se rige por la Constitución de la República del Ecuador, la Ley Orgánica de Educación Superior, su Reglamento, otras leyes conexas, el presente Estatuto, los Reglamentos $y$ Resoluciones que expidan el Consejo de Educación Superior; el Consejo de Evaluación, Acreditación y Aseguramiento de la Calidad de la Educación Superior; y, la Secretaría Nacional de Educación Superior, Ciencia, Tecnología e Innovación y la Universidad (Estatuto de la Universidad Nacional de Chimborazo, 2013, p. 1).

Según el Estatuto de la Universidad Nacional de Chimborazo (2013), la estructura del gobierno de la Universidad está compuesta por: el consejo universitario, el rector, el vicerrector académico, el vicerrector de posgrado e investigación, el vicerrector administrativo, el consejo general académico, el consejo de investigación, los consejos directivos de grado y posgrado, los decanos, y los subdecanos. 
La Universidad tiene cuatro facultades y oferta un total de 31 carreras académicas. Además, cuenta con un total de 7761 estudiantes, 694 docentes y 745 personas que trabajan como personal administrativo (tabla 1).

Tabla 1

Facultades, Carreras y Número de estudiantes de la Universidad Nacional de Chimborazo

\begin{tabular}{lcc}
\hline \multicolumn{1}{c}{ Facultades } & $\begin{array}{c}\mathbf{N}^{\circ} \text { de } \\
\text { carreras }\end{array}$ & $\begin{array}{c}\mathbf{N}^{\circ} \text { de } \\
\text { estudiantes }\end{array}$ \\
\hline $\begin{array}{l}\text { Facultad de Ciencias de la Salud } \\
\text { Facultad de Ciencias de }\end{array}$ & 7 carreras & 2539 \\
$\begin{array}{l}\text { Educación } \\
\text { Facultad de Ingeniería }\end{array}$ & 8 carreras & 1179 \\
$\begin{array}{l}\text { Facultad de Ciencias Políticas y } \\
\text { Administrativas }\end{array}$ & 5 carreras & 2089 \\
Total & 31 carreras & 7761 \\
\hline
\end{tabular}

Fuente: Sistema Académico SICOA, 2019

La Instituciones de Educación Superior, para la elaboración de su presupuesto anual es indispensable que determine los costos de la prestación de los servicios educativos, en este sentido los resultados de la investigación muestran que los métodos de costo utilizados para valorar los servicios en la universidad son, el costo total en un $47,5 \%$, el costo de la calidad en un $47,5 \%$, y costos por procesos en un $42.10 \%$ lo que quiere decir, que la institución utiliza un sistema de costos tradicional, no cuenta con un sistema específico, viene utilizando estos métodos y no el método de costo $\mathrm{ABC}$; sin embargo, se identifica que la universidad determina el costo real de los servicios, como se presenta en la tabla 2.

La implantación de un método de costos, de acuerdo a los resultados de la investigación, implica superar barreras como: la falta de especialistas en costos al interior de la universidad, la falta de actualización de conocimientos en técnicas vigentes de los directivos en costos, el análisis de datos con reporte de información, el trabajo extra y un personal capacitado. Esto da a entender que el beneficio de la adopción de un método de costos en la universidad a pesar de existir barreras puede ser muy importante para la determinación de costos reales.

De igual forma, en la tabla 3 se identifica que los objetivos del sistema de contabilidad de costos son: reducir los costos de los servicios educativos un $37,5 \%$, suministrar información para las funciones operativas un $25 \%$, conocer los costos un 25\%, para control un $15,2 \%$ y suministrar información para la toma de decisiones un 15,2\%. Significa que la universidad implementa un sistema de costos principalmente con el objetivo de reducir y conocer los costos, a fin de suministrar información a funciones operativas y permitir la toma de decisiones.

Con relación a la implementación del sistema $\mathrm{ABC}$ en la Universidad, los resultados de la investigación muestran que; medianamente importante, no se tiene implementado el sistema de costo $A B C$, pero se evalúa la posibilidad

Tabla 2

Método de costo utilizado en la universidad

\begin{tabular}{|c|c|c|c|c|c|c|}
\hline $\begin{array}{l}\text { Qué tipo de método de costos utiliza en la determi- } \\
\text { nación del costos de la prestación de los servicios }\end{array}$ & Si & $\begin{array}{c}\text { No pero está } \\
\text { siendo Instalada }\end{array}$ & $\begin{array}{l}\text { No, pero estamos } \\
\text { pensando }\end{array}$ & No & $\begin{array}{l}\text { No lo } \\
\text { conozco }\end{array}$ & Total \\
\hline Costo de calidad & 47,5 & 2,5 & 0 & 12,5 & 37,5 & 100 \\
\hline Costo Total & 47,5 & 2,5 & 0 & 12,5 & 37,5 & 100 \\
\hline Costo por procesos & 42,1 & 2,6 & 5,3 & 13,2 & 36,8 & 100 \\
\hline
\end{tabular}

Fuente: Elaboración propia

Tabla 3

Objetivos de un sistema contable de costos

\begin{tabular}{|c|c|c|c|c|c|c|c|}
\hline Objetivos del sistema de contabilidad de costos & $\begin{array}{c}\text { No es } \\
\text { importante } \\
\text { (1) }\end{array}$ & $\begin{array}{c}\text { Poco } \\
\text { importante } \\
\text { (2) }\end{array}$ & $\begin{array}{c}\text { Neutral } \\
(3)\end{array}$ & $\begin{array}{c}\text { Importante } \\
\text { (4) }\end{array}$ & $\begin{array}{c}\text { Muy } \\
\text { importante } \\
\text { (5) }\end{array}$ & Total & Moda \\
\hline Conocer Costos & 12,5 & 9,4 & 25 & 28,1 & 25 & 100 & 4 \\
\hline Control & 15,2 & 21,2 & 24,2 & 24,2 & 15,2 & 100 & 3 \\
\hline Suministrar información para la toma de decisiones & 24,2 & 18,2 & 24,2 & 18,2 & 15,2 & 100 & 1 \\
\hline Suministrara información para funciones operativas & 12,5 & 25 & 18,8 & 18,8 & 25 & 100,1 & 2 \\
\hline Reducción Costos & 28,1 & 9,4 & 15,6 & 9,4 & 37,5 & 100 & 5 \\
\hline
\end{tabular}

Fuente: Elaboración propia 
en un 30,30\%; sumamente importante el 30,60\% manifiestan que no se ha planteado por el momento implementar y en un $44,4 \%$ se analiza la posibilidad de implementar. De esta manera, el sistema ABC se considera que es sumamente importante, a pesar de que no se ha implementado por el momento; sin embargo, se viene evaluando y analizando la posibilidad como se muestra en la tabla 4. Por tanto, existe una alta posibilidad de que el sistema de costos $\mathrm{ABC}$ sea implementado en la UNACH y en diferentes universidades de Latinoamerica.

Para demostrar si el sistema de costos $\mathrm{ABC}$ no influye significativamente en la determinación de los costos reales en la Universidad Nacional de Chimborazo (hipótesis 1) se procedió a la aplicación del modelo de regresión lineal, representada de la siguiente manera:
$\mathrm{Y}=\mathrm{bo}+\mathrm{b} 1 \mathrm{x} 1+\mathrm{b} 2 \times 2+\mathrm{b} 3 \times 3+\ldots+\mathrm{bnxn}+\mathrm{u}$

Dónde: Sistema de costos $\mathrm{ABC}=\mathrm{b} 0+\mathrm{b} 1 \mathrm{x}$ Conocer costos

El resultado muestra que no existe un grado de dependencia (Sig. 0,321) y R2=0,038 aunque existe una relación de $19,4 \%(r=2)$, como se observa en la tabla 5.

El grado de influencia del modelo de costos $\mathrm{ABC}$ en la determinación de los costos reales es relativamente bajo, por tanto, no es determinante.

También, a fin de demostrar la hipótesis $\mathrm{H} 2$ = si los procedimientos y actividades relacionados a la gestión, docencia e investigación influyen significativamente en la determinación de los costos reales en la UNACH, se procedió a la aplicación el modelo factorial y luego la aplicación

Tabla 4

Implementación del sistema $A B C$ en la Universidad Nacional de Chimborazo

\begin{tabular}{|c|c|c|c|c|c|c|c|c|c|c|c|c|}
\hline \multirow[b]{2}{*}{$\begin{array}{l}\text { Alternativas } \\
\text { de respuestas }\end{array}$} & \multicolumn{4}{|c|}{$\begin{array}{l}\text { No se tiene pero se evalúa la } \\
\text { posibilidad del } A B C\end{array}$} & \multicolumn{4}{|c|}{$\begin{array}{c}\text { No se ha planteado por el } \\
\text { momento }\end{array}$} & \multicolumn{4}{|c|}{$\begin{array}{l}\text { Se analiza la posibilidad de } \\
\text { implementar }\end{array}$} \\
\hline & 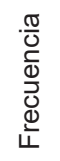 & $\begin{array}{l}\frac{0}{\pi} \\
\stackrel{0}{0} \\
\frac{0}{0} \\
\frac{0}{0} \\
0\end{array}$ & $\begin{array}{l}\frac{0}{\pi} \\
\stackrel{0}{\frac{\pi}{0}} \\
\frac{0}{0} \\
\frac{0}{0} \\
0 \\
0\end{array}$ & 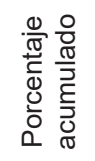 & 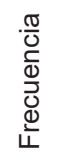 & $\begin{array}{l}\frac{0}{\pi} \\
\frac{\pi}{0} \\
\frac{0}{0} \\
0 \\
0\end{array}$ & 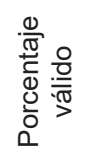 & 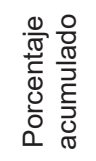 & 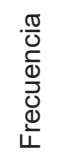 & $\begin{array}{l}\frac{0}{\pi} \\
\stackrel{0}{0} \\
\frac{0}{0} \\
\frac{0}{0} \\
0\end{array}$ & 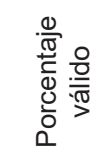 & 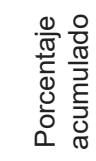 \\
\hline $\begin{array}{l}\text { No se toma en } \\
\text { cuenta }\end{array}$ & 5 & 8,9 & 15,2 & 15,2 & 5 & 8,9 & 13,9 & 13,9 & 3 & 5,4 & 8,3 & 8,3 \\
\hline $\begin{array}{l}\text { Poco } \\
\text { importante }\end{array}$ & 1 & 1,8 & 3,0 & 18,2 & 3 & 5,4 & 8,3 & 22,2 & 1 & 1,8 & 2,8 & 11,1 \\
\hline $\begin{array}{l}\text { Medianamente } \\
\text { Importante }\end{array}$ & 10 & 17,9 & 30,3 & 48,5 & 6 & 10,7 & 16,7 & 38,9 & 6 & 10,7 & 16,7 & 27,8 \\
\hline $\begin{array}{l}\text { Sumamente } \\
\text { Importante }\end{array}$ & 9 & 16,1 & 27,3 & 75,8 & 11 & 19,6 & 30,6 & 69,4 & 16 & 28,6 & 44,4 & 72,2 \\
\hline Indispensable & 8 & 14,3 & 24,2 & 100,0 & 11 & 19,6 & 30,6 & 100,0 & 10 & 17,9 & 27,8 & 100,0 \\
\hline Total & 33 & 58,9 & 100,0 & & 36 & 64,3 & 100,0 & & 36 & 64,3 & 100,0 & \\
\hline Perdidos & 23 & 41,1 & & & 20 & 35,7 & & & 20 & 35,7 & & \\
\hline Total & 56 & 100,0 & & & 56 & 100 & & & 56 & 100,0 & & \\
\hline
\end{tabular}

Fuente: Elaboración propia

Tabla 5

Resumen del modelo de costos $A B C$

\begin{tabular}{ccccc|cccc}
\hline Modelo & $\mathbf{R}$ & $\mathbf{R}$ cuadrado & $\begin{array}{c}\mathbf{R} \text { cuadrado } \\
\text { ajustado }\end{array}$ & $\begin{array}{c}\text { Error estándar de } \\
\text { la estimación }\end{array}$ & $\begin{array}{c}\text { Cambio de cuadrado } \\
\text { de } \mathrm{R}\end{array}$ & Cambio en $\mathrm{F}$ & $\begin{array}{c}\text { Sig. Cambio } \\
\text { en } \mathrm{F}\end{array}$ & $\begin{array}{c}\text { Sig. } \\
, 321 \mathrm{~b}\end{array}$ \\
\hline $\mathbf{1}$ &, $194 \mathrm{a}$ & 0,038 & 0,001 & 0,41769 & 0,038 & 1,022 & 0,321 \\
\hline
\end{tabular}

Fuente: Elaboración propia 
de la regresión lineal para identificar si estos influyen. El modelo factorial considera un conjunto de $\mathrm{p}$ variables observadas como $\mathrm{x}=(\mathrm{x} 1, \mathrm{x} 2, \mathrm{x} 3, \ldots \mathrm{xp})$ que se relacionan con un número de variables $\mathrm{f} 1, \mathrm{f} 2, \mathrm{f} 3$, fk, dando como resultado $\mathrm{k}<\mathrm{p}$. Los resultados de la factorización, muestran que existen procedimientos relacionados con los 1) procesos gobernantes, 2) gestión académica, 3) gestión de admisión y nivelación, 4) gestión de la investigación, 5) desarrollo y difusión de la investigación, 6) vinculación con la sociedad, 7) infraestructura equipos y acervos bibliográficos,8) proceso de asesoría, 9) proceso de la gestión administrativa. Estos a su vez, son explicados por un conjunto de actividades que involucra cada procedimiento, como se presenta en la tabla 6 .

De este modo, los factores más significativos están relacionados con los procedimiento y actividades, como: 1) Las actividades de procesos gobernantes, es importante en un $66,8 \%$ y está relacionado a la actividad de direccionamiento estratégico Institucional en un $88,4 \%$ ); 2) El procedimiento de gestión académica, importante en un 66,75\%, relacionada a Actividad de orientación y acompañamiento a través de tutorías presenciales virtuales, individuales y grupales en un 90,59\%; 3) El procedimiento de gestión de admisión y nivelación, importante en un 75,34\%, relacionado con distintas actividades, 4) Procedimiento de gestión de investigación, importante en un $76,98 \%$, siendo las actividades más importante: con las actividades de Organización de la investigación en un 92,12\%; 5) Procedimiento del desarrollo y difusión de la investigación; este procedimiento está relacionado con distintas actividades en un $83,89 \%$; 6) Procedimiento de gestión de vinculación, importante en un 76,20\%; 7) Procedimiento de gestión de infraestructura, equipos y acervos bibliográficos, importante en un 79,27\%; 8) Procedimiento del proceso de asesoría; importante en un 76,88\%; 9) Procedimiento del proceso de gestión administrativa; importante en un $73,87 \%$ relacionado con actividades más importantes, como: la actividad de gestión financiera en un 93,72\%. Estos resultados permiten demostrar que las actividades y procedimientos están relacionados a las funciones de gestión, docencia e investigación y que las actividades identificadas son los determinantes de los procedimientos para que a partir de ellas se pueda determinar los costos, a través del uso del método de costos $\mathrm{ABC}$. Además, el sistema de costos $\mathrm{ABC}$ permite identificar procesos y actividades que pueden ser considerados para la determinación de los costos reales.

\section{DISCUSIÓN}

El método de costeo ABC puede determinar los costos de los servicios de manera eficiente y eficaz; optimizar los recursos económicos, proporcionar información

Tabla 6

Procedimientos y actividades para la aplicación del método de costos abc

\begin{tabular}{|c|c|c|c|c|c|c|c|c|}
\hline Actividades & Media & $\begin{array}{l}\text { No se toma } \\
\text { encuentra }\end{array}$ & $\begin{array}{l}\text { Poco } \\
\text { importante }\end{array}$ & $\begin{array}{l}\text { Mediana- } \\
\text { mente } \\
\text { Importante }\end{array}$ & $\begin{array}{l}\text { Sumamente } \\
\text { Importante }\end{array}$ & Indispensable & KMO & $\begin{array}{l}\text { Factor } \\
\text { explicación }\end{array}$ \\
\hline Actividades de procesos gobernantes & 4,2 & 2,6 & 1,3 & 6,5 & 35,7 & 53,9 & 0,628 & 0,668 \\
\hline Actividades de gestion academica & 4,28 & 1,57 & 2,15 & 7,65 & 44,14 & 44,48 & 0,906 & 0,6675 \\
\hline $\begin{array}{l}\text { Actividades del proceso de la } \\
\text { gestión de admisión y nivelación }\end{array}$ & 4,18 & 4,96 & 0 & 6,92 & 48,49 & 39,63 & 0,5 & 0,7534 \\
\hline $\begin{array}{l}\text { Actividades de gestión de la } \\
\text { investigación }\end{array}$ & 4,33 & 2,24 & 0,99 & 5,2 & 44,29 & 47,28 & 0,8995 & 0,7698 \\
\hline $\begin{array}{l}\text { Actividades de desarrollo y difusión de } \\
\text { la investigación }\end{array}$ & 4,32 & 1,97 & 0 & 7,36 & 44,94 & 45,74 & 0,779 & 0,8389 \\
\hline Actividades de vinculación & 4,31 & 4,6 & 0 & 6,26 & 37,94 & 51,2 & 0,786 & 0,762 \\
\hline $\begin{array}{l}\text { Actividades de gestión de la } \\
\text { infraestructura, laboratorios, equipos } \\
\text { y acervos bibliográficos }\end{array}$ & 4,4 & 2 & 0 & 3,99 & 43,76 & 50,26 & 0,893 & 0,7927 \\
\hline $\begin{array}{l}\text { Actividades de los procesos } \\
\text { habilitantes asesoria }\end{array}$ & 4,43 & 2,84 & 0 & 2,59 & 47,76 & 46,81 & 0,897 & 0,7688 \\
\hline
\end{tabular}

Fuente: Elaboración propia 
confiable y generar un valor añadido a las actividades a fin de facilitar procesos de decisión. Esto significa que puede ser aplicado en instituciones de carácter educativo; como es la Universidad Nacional de Chimborazo y otras universidades de los distintos países de América Latina. El sistema de costos está relacionado con una serie de pasos y su aplicación con un conjunto de procedimientos y actividades que influyen en la aplicación o no de un sistema de costos $\mathrm{ABC}$ en las universidades, como también menciona Boschin y Metz (2009). Coincidimos con Ficco (2011), donde el método de costos ABC es una herramienta que permite tener información de costos de calidad, tomar decisiones, y desarrollar la gerencia estratégica de costos. Del Carpio (2007), menciona que los procesos para la aplicación del método de costos $\mathrm{ABC}$ está compuesto por una serie de actividades que permiten organizar el trabajo de los costos, como: 1) La elaboración de un mapa de procesos, actividades, recursos y servicios institucionales; 2) Identificación de los costos directos e indirectos en los que se incurren; 3 ) Identificación de la relación de los productos que oferta la institución; 4) Identificación de los procesos y la distribución de los recursos, tomando en consideración que en cada proceso se debe distribuir recursos; 5) Identificación de las actividades (procesos gobernantes, de formación, docencia, investigación, vinculación, infraestructura, laboratorios, bibliotecas, y servicios); 6) Asignación de los costos de los recursos a las actividades. (Proceso que se lo realiza por medio de inductores o tasas de asignación); y 7) Análisis de los resultados, mediante el diseño de herramientas que permita levantar la información con el fin de generar reportes que admita relacionar las actividades con los servicios que prestan las Instituciones de Educación Superior. En tal caso, los resultados de la investigación permiten confirmar que la determinación de los costos depende de los procedimientos relacionados a las funciones de gestión, docencia e investigación y a actividades que implican el desarrollo de cada uno de ellos.

\section{REFERENCIAS BIBLIOGRÁFICAS}

Arellano, O., Quispe, G., Ayaviri, D., \& Escobar, F. (2017). Estudio de la Aplicación del Método de Costos ABC en las Mypes del Ecuador. Investigación Altoandinas, 19(1), 33-46. Recuperado de: http://dx.doi. org/10.18271/ria.2016.253

Boschin, M., \& Metz, N. (2009). Gestión de costos en Instituciones Educativas. Revista del Instituto Internacional de Costos (5), 563-587.

Calderón, J. (2014). Contabilidad de Costos I: Teoría Práctica (Quinta edición). Lima: JCM Editores.

Chambergo, G. (2014). Contabilidad de Costos para la Toma de Decisiones: Aplicación Práctica. Lima: Actua- lidad empresarial Pacífica Editores.

Del Carpio, J. (2007). Aplicación del costeo basado en actividades en las Universidades. Industrial Data Revista de Investigación, 10(2), 26-29.

Del Rio, R., Rodríguez, V., García, T., \& Sánchez, J. (2018). Diseño del mapa de actividades por el modelo de costes $\mathrm{ABC}$ para los departamentos universitarios. Cuadernos de Gestión, 19(2), 159-184.

Fernandes, V., Barbosa, A., Mendes, E., \& Del Canto, E. (2014). Sistema de costos ABC en la gestión de los hospitales: una reflexión. Ingeniería y Sociedad UC, 10(1), 78-87.

Ficco, C. (2011). El costeo Basado en la Actividad en las Universidades: Una herramienta para la gestión estratégica y la creación de valor. SaberEs, (3), 27-46.

García, D., Marín, S., \& Martínez, F. (2006). La contabilidad de costos y rentabilidad en la Pyme. Contaduría y Administración, (218), 39-59.

García, J. (2008). Contabilidad de costos (3ra. ed.). México D.F.: McGraw-Hill Interamericana.

Hansen, D., \& Mowen, M. (2007). Administración de Costos Contabilidad y Control (5ta. ed.). México D.F.: CENGAGE Learning.

Horngren, C. T., Datar, S. M., \& Rajan, M. V. (2012). Contabilidad de costos. Un enfoque gerencial (Decimocuarta Edición ed.). México, México: Pearson Educación.

Kaplan, R. (1984). La evolución de la contabilidad de gestión. The Accounting Review, 59(3), 390-418.

López, M., \& Rodríguez, J. (2018). Particularidades del Costo en las Universidades. Apuntes contables (21), 103-115. Recuperado de: https://doi. org/10.18601/16577175.n21.08.

Navarro, J., Martínez, L., Castilla, J., \& Hernández, E. (2006). Coste de las técnicas de reproducción asistida en un hospital público. Gac. Sanit, 20(5), 382-391.

Pérez, G., Vergara, J., Moreno, J., Vergara, G., \& Rodríguez, C. (2008). El sistema de costos por actividad en el sector educativo. Libre Empresa, 5(1), 95-120.

Tinjaca, D. (2005). Modelo de un sistema de costeo por actividades para la universidad distrital. Bogotá: Universidad Distrital Francisco José de Caldas.

Universidad Nacional de Chimborazo. (2012). Plan estratégico de desarrollo institucional 2012 - 2016. 
Riobamba: Unach. Resolución Nro. 0337-HCU-1710-2012.

Universidad Nacional de Chimborazo. (2013). Estatuto de la Universidad Nacional de Chimborazo. Riobamba:
Unach. Recuperado de: http://www.unach.edu.ec/images/pdf/noticias/octubre_2013/estatuto_unach.pdf

Uribe, R. (2011). Costos para la toma de decisiones. Primera edición. Bogotá: McGrawHill. 